PROCEEDINGS OF THE

AMERICAN MATHEMATICAL SOCIETY

Volume 139, Number 2, February 2011, Pages 421-434

S 0002-9939(2010)10689-5

Article electronically published on September 21, 2010

\title{
THE LIMIT OF $\mathbb{F}_{p}$-BETTI NUMBERS OF A TOWER OF FINITE COVERS WITH AMENABLE FUNDAMENTAL GROUPS
}

\author{
PETER LINNELL, WOLFGANG LÜCK, AND ROMAN SAUER \\ (Communicated by Brooke Shipley)
}

\begin{abstract}
We prove an analogue of the Approximation Theorem of $L^{2}$-Betti numbers by Betti numbers for arbitrary coefficient fields and virtually torsionfree amenable groups. The limit of Betti numbers is identified as the dimension of some module over the Ore localization of the group ring.
\end{abstract}

\section{INTRODUCTION}

A residual chain of a group $G$ is a sequence $G=G_{0} \supset G_{1} \supset G_{2} \supset \cdots$ of normal subgroups of finite index such that $\bigcap_{i>0} G_{i}=\{e\}$. The $n$-th $L^{2}$-Betti number of any finite free $G$-CW complex $X$ is the limit of the $n$-th Betti numbers of $G_{i} \backslash X$ normalized by the index $\left[G: G_{i}\right]$ for $i \rightarrow \infty$ [11. If we instead consider Betti numbers $b_{n}\left(G_{i} \backslash X ; k\right)$ with respect to a field of characteristic $p>0$, the questions as to whether the limit exists, what it is, and whether it is independent of the residual chain are completely open for arbitrary residually finite $G$.

For $G=\mathbb{Z}^{k}$ and every field $k$ Elek showed that $\lim _{i \rightarrow \infty} b_{n}\left(G_{i} \backslash X ; k\right)$ exists and expresses it in terms of the entropy of $G$-actions on the Pontrjagin duals of finitely generated $k G$-modules 4 - his techniques play an important role in this paper (see Section [1.3). It was observed in [1, Theorem 17] that the mere convergence of the right hand side of (i) in Theorem 0.2 for every amenable $G$ and every field $k$ follows from a general convergence principle for subadditive functions on amenable groups [9] and a theorem by Weiss [18.

The main purpose of this paper is to determine the $\operatorname{limit}_{\lim _{i \rightarrow \infty}} b_{n}\left(G_{i} \backslash X ; k\right)$ in algebraic terms for a large class of amenable groups including virtual torsionfree elementary amenable groups. This makes the limit computable by homological techniques; see e.g., the spectral sequence argument of Example 6.3.

More precisely, the limit will be expressed in terms of the Ore dimension. The group ring $k G$ of a torsionfree amenable group satisfying the zero-divisor conjecture fulfills the Ore condition with respect to the subset $S=k G-\{0\}$ 12, Example 8.16 on page 324]; we will review the Ore localization in Subsection 1.1. The Ore

Received by the editors March 1, 2010.

2010 Mathematics Subject Classification. Primary 16U20, 55P99.

Key words and phrases. Amenability, Ore localization, Betti numbers.

The authors thank the HIM at Bonn for its hospitality during the trimester program "Rigidity" in the fall of 2009, when this paper was written. This work was financially supported by the Leibniz-Preis of the second author. 
localization $S^{-1} k G$ is a skew field containing $k G$. Therefore the following definition makes sense:

Definition 0.1 (Ore dimension). Let $G$ be a torsionfree amenable group such that $k G$ contains no zero-divisors. The Ore dimension of a $k G$-module $M$ is defined by

$$
\operatorname{dim}_{k G}^{\text {Ore }}(M)=\operatorname{dim}_{S^{-1} k G}\left(S^{-1} k G \otimes_{k G} M\right) .
$$

The following theorem is our main result. We will prove a more general version, including virtually torsionfree groups, in Section 5 .

Theorem 0.2. Let $k$ be a field. Let $G$ be a torsionfree amenable group for which $k G$ has no zero-divisors 1 Let $\left(G_{n}\right)_{n \geq 0}$ be a residual chain of $G$. Then:

(i) Consider a finitely presented $k G$-module $M$. Then

$$
\operatorname{dim}_{k G}^{\text {Ore }}(M)=\lim _{n \rightarrow \infty} \frac{\operatorname{dim}_{k}\left(k \otimes_{k G_{n}} M\right)}{\left[G: G_{n}\right]} .
$$

(ii) Consider a finite free $k G$-chain complex $C_{*}$. Then we get for all $i \geq 0$

$$
\operatorname{dim}_{k G}^{\text {Ore }}\left(H_{i}\left(C_{*}\right)\right)=\lim _{n \rightarrow \infty} \frac{\operatorname{dim}_{k}\left(H_{i}\left(k \otimes_{k G_{n}} C_{*}\right)\right)}{\left[G: G_{n}\right]} .
$$

(iii) Let $X$ be a finite free $G$-CW-complex. Then we get for all $i \geq 0$

$$
\operatorname{dim}_{k G}^{\text {Ore }}\left(H_{i}(X)\right)=\lim _{n \rightarrow \infty} \frac{\operatorname{dim}_{k}\left(H_{i}\left(G_{n} \backslash X ; k\right)\right)}{\left[G: G_{n}\right]} .
$$

Remark 0.3 (Fields of characteristic zero). Let $G$ be a group with a residual chain $\left(G_{n}\right)_{n \geq 0}$, and let $M$ be a finitely presented $k G$-module. Then the Approximation Theorem for $L^{2}$-Betti numbers says that

$$
\operatorname{dim}_{\mathcal{N}(G)}\left(\mathcal{N}(G) \otimes_{k G} M\right)=\lim _{n \rightarrow \infty} \frac{\operatorname{dim}_{k}\left(k \otimes_{k G_{n}} M\right)}{\left[G: G_{n}\right]},
$$

provided $k$ is an algebraic number field. Here $\mathcal{N}(G)$ is the group von Neumann algebra and $\operatorname{dim}_{\mathcal{N}(G)}$ is the von Neumann dimension. See [1] for $k=\mathbb{Q}$ and [3] for the general case.

Let $k$ be a field of characteristic zero and let $u=\sum_{g \in G} x_{g} \cdot g \in k G$ be an element. Let $F$ be the finitely generated field extension of $\mathbb{Q}$ given by $F=\mathbb{Q}\left(x_{g} \mid g \in G\right) \subset k$. Then $u$ is already an element in $F G$. The field $F$ embeds into $\mathbb{C}$ : since $F$ is finitely generated, it is a finite algebraic extension of a transcendental extension $F^{\prime}$ of $\mathbb{Q}$ ([8], Theorem 1.1 on p. 356) and $F^{\prime}$ has finite transcendence degree over $\mathbb{Q}$. Since the transcendence degree of $\mathbb{C}$ over $\mathbb{Q}$ is infinite, there exists an embedding $F^{\prime} \hookrightarrow \mathbb{C}$ induced by an injection of a transcendence basis of $F^{\prime} / \mathbb{Q}$ into a transcendence basis $\mathbb{C} / \mathbb{Q}$, which extends to $F \hookrightarrow \mathbb{C}$ because $\mathbb{C}$ is algebraically closed. This reduces the case of fields of characteristic zero to the case $k=\mathbb{C}$. In [6] Elek proved (0.4) for amenable $G$ and $k=\mathbb{C}$ (see also [13]).

Moreover, if $G$ is a torsionfree amenable group such that $\mathbb{C} G$ contains no zerodivisors and $k$ is a field of characteristic zero, then

$$
\operatorname{dim}_{\mathcal{N}(G)}\left(\mathcal{N}(G) \otimes_{k G} M\right)=\operatorname{dim}_{k G}^{\text {Ore }}(M)
$$

\footnotetext{
${ }^{1}$ This assumption is satisfied if $G$ is torsionfree elementary amenable. See Remark 1.1
} 
This follows from [12, Theorem 6.37 on page 259, Theorem 8.29 on page 330, Lemma 10.16 on page 376, and Lemma 10.39 on page 388]. In particular, Theorem 0.2 follows for $k$ of characteristic zero. So the interesting new case is the one of a field of prime characteristic.

\section{Review of Ore localization and Elek's dimension function}

1.1. Ore localization. We review the Ore localization of rings. For proofs and more information the reader is referred to [17]. Consider a torsionfree group $G$ and a field $k$. Let $S$ be the set of non-zero-divisors of $k G$. This is a multiplicatively closed subset of $k G$ and contains the unit element of $k G$. Suppose that $k G$ satisfies the Kaplansky Conjecture or zero-divisor conjecture, i.e., $S=k G-\{0\}$. Further assume that $S$ satisfies the left Ore condition, i.e., for $r \in k G$ and $s \in S$ there exists $r^{\prime} \in k G$ and $s^{\prime} \in S$ with $s^{\prime} r=r^{\prime} s$. Then we can consider the Ore localization $S^{-1} k G$. Recall that every element in $S^{-1} k G$ is of the form $s^{-1} \cdot r$ for $r \in k G$ and $s \in S$ and that $s_{0}^{-1} \cdot r_{0}=s_{1}^{-1} \cdot r_{1}$ holds if and only there exists $u_{0}, u_{1} \in R$ satisfying $u_{0} r_{0}=u_{1} r_{1}$ and $u_{0} s_{0}=u_{1} s_{1}$. Addition is given on representatives by $s_{0}^{-1} r_{0}+s_{1}^{-1} r_{1}=t^{-1}\left(c_{0} r_{0}+c_{1} r_{1}\right)$ for $t=c_{0} s_{0}=c_{1} s_{1}$. Multiplication is given on representatives by $s_{0}^{-1} r_{0} \cdot s_{1}^{-1} r_{1}=\left(t s_{0}\right)^{-1} c r_{1}$, where $c s_{1}=t r_{0}$. The zero element is $e^{-1} \cdot 0$, and the unit element is $e^{-1} \cdot e$. The Ore localization $S^{-1} k G$ is a skew field, and the canonical map $k G \rightarrow S^{-1} k G$ sending $r$ to $e^{-1} \cdot r$ is injective. The functor $S^{-1} k G \otimes_{k G}-$ is exact.

Remark 1.1 (The Ore condition for group rings). If a torsionfree amenable group $G$ satisfies the Kaplansky Conjecture, i.e., $k G$ contains no zero-divisor, then for $S=$ $k G-\{0\}$ the Ore localization $S^{-1} k G$ exists and is a skew field [12, Example 8.16 on page 324]. Every torsionfree elementary amenable group satisfies the assumptions above for all fields $k$, Theorem 1.2; 10, Theorem 2.3]. If the group $G$ contains the free group of rank two as a subgroup, then the Ore condition is never satisfied for $k G$ [10, Proposition 2.2].

From the previous remark and the discussion above we obtain:

Theorem 1.2. Let $G$ be a torsionfree amenable group such that $k G$ contains no zero-divisors. Then the Ore dimension $\operatorname{dim}_{k G}^{\text {Ore }}$ has the following properties:

(i) $\operatorname{dim}_{k G}^{\text {Ore }}(k G)=1$.

(ii) For any short exact sequence of $k G$-modules $0 \rightarrow M_{0} \rightarrow M_{1} \rightarrow M_{2} \rightarrow 0$ we get

$$
\operatorname{dim}_{k G}^{\text {Ore }}\left(M_{1}\right)=\operatorname{dim}_{k G}^{\text {Ore }}\left(M_{0}\right)+\operatorname{dim}_{k G}^{\text {Ore }}\left(M_{2}\right) .
$$

1.2. Crossed products, Goldie rings, and the generalized Ore localization. Throughout, let $G$ be a group and let $k$ be a skew field.

Let $R$ be a ring. The notion of a crossed product generalizes the one of a group ring. A crossed product $R * G=R *_{c, \tau} G$ is determined by maps $c: G \rightarrow \operatorname{aut}(R)$ and $\tau: G \times G \rightarrow R^{\times}$such that, roughly speaking, $c$ is a homomorphism up to the 2-cocycle $\tau$. We refer to the survey [12, 10.3 .2 on p. 398] for details. If $G$ is an extension of $H$ by $Q$, then the group ring $k G$ is isomorphic to a crossed product $k H * Q$. Some results in this paper are formulated for crossed products, although we only need the case of group rings for Theorem 0.2 So the reader may think of group rings most of the time. However, crossed products show up naturally, e.g., in proving that the virtual Ore dimension (5.1) is well defined. 
We recall the following definition.

Definition 1.3. A ring $R$ is left Goldie if there exists $d \in \mathbb{N}$ such that every direct sum of non-zero left ideals of $R$ has at most $d$ summands and the left annihilators $a(x)=\{r \in R ; r x=0\}, x \in R$, satisfy the maximum condition for ascending chains. A ring $R$ is prime if for any two ideals $A, B$ in $R, A B=0$ implies $A=0$ or $B=0$.

The subgroup of $G$ generated by its finite normal subgroups will be denoted by $\Delta^{+}(G)$. Then $\Delta^{+}(G)$ is also the set of elements of finite order which have only finitely many conjugates. We need the following three results:

Lemma 1.4 ([15, Corollary 5 of Lecture 4]). If $\Delta^{+}(G)=1$, then $k * G$ is prime.

Theorem 1.5 ([14, Theorem 4.10 on p. 456]). The set of non-zero-divisors in a prime left Goldie ring satisfies the Ore condition. The Ore localization $S^{-1} R$ is isomorphic to $\mathrm{M}_{d}(D)$ for some $d \in \mathbb{N}$ and some skew field $D$.

Theorem 1.6. If $G$ is amenable and $k * G$ is a domain, then $k * G$ is a prime left Goldie ring. If $G$ is an elementary amenable group such that the orders of the finite subgroups are bounded, then $k * G$ is left Goldie.

Proof. If $G$ is amenable and $k * G$ is a domain, then $k * G$ satisfies the Ore condition [3, Theorem 6.3]. Thus its Ore localization with respect to $S=k * G-\{0\}$ is a skew field. By [14, Theorem 4.10 on p. 456] $k * G$ is a prime left Goldie ring. The second assertion is taken from [7, Proposition 4.2].

Next we extend the definition of Ore dimension to prime left Goldie rings. Let $R$ be such a ring. The functor $S^{-1} R \otimes_{R}-$ will still be exact [17, Proposition II.1.4 on page 51]. If $M$ is a left $R$-module, then $S^{-1} R \otimes_{R} M$ will be a direct sum of $n$ irreducible $S^{-1} R$-modules for some non-negative integer $n$, and then the (generalized) Ore dimension of $M$ is defined as

$$
\operatorname{dim}_{R}^{\text {Ore }}(M)=\frac{n}{d} .
$$

Since $S^{-1} R \cong \mathrm{M}_{d}(D)$ (Theorem 1.5) and $\mathrm{M}_{d}(D)$ decomposes into $d$ copies of the irreducible module $D^{d}$, we have $\operatorname{dim}_{R}^{\text {Ore }}(R)=1$.

1.3. Elek's dimension function. Throughout this subsection let $G$ be a finitely generated amenable group. We review Elek's definition [5] of a dimension function $\operatorname{dim}_{k G}^{\text {Elek }}$ for finitely generated $k G$-modules.

Fix a finite set of generators and equip $G$ with the associated word metric $d_{G}$. A Følner sequence $\left(F_{n}\right)_{n \geq 0}$ is a sequence of finite subsets of $G$ such that for any fixed $R>0$ we have

$$
\lim _{n \rightarrow \infty} \frac{\left|\partial_{R} F_{n}\right|}{\left|F_{n}\right|}=0
$$

where $\partial_{R} F_{n}=\left\{g \in G \mid d\left(g, F_{k}\right) \leq R\right.$ and $\left.d\left(g, G \backslash F_{k}\right) \leq R\right\}$.

Let $k$ be an arbitrary skew field endowed with the discrete topology and let $\mathbb{N}$ denote the positive integers $\{1,2, \ldots\}$. Let $n \in \mathbb{N}$. We equip the space of functions $\operatorname{map}\left(G, k^{n}\right)=\prod_{g \in G} k^{n}$ with the product topology, which is the same as the topology of pointwise convergence. The natural right $G$-action on $\operatorname{map}\left(G, k^{n}\right)$ is defined by

$$
(\phi g)(x)=\phi\left(x g^{-1}\right) \text { for } g, x \in G, \phi \in \operatorname{map}\left(G, k^{n}\right) .
$$


Also $\operatorname{map}\left(G, k^{n}\right)$ is a right $k$-vector space by defining $(\phi k)(x)=\phi(x) k$. For any subset $S \subset G$ and any subset $W \subset \operatorname{map}\left(G, k^{n}\right)$, let

$$
\left.W\right|_{S}=\left\{f: S \rightarrow k^{n} \mid \exists g \in W \text { with }\left.g\right|_{S}=f\right\} .
$$

A right $k$-linear subspace $V \subset \operatorname{map}\left(G, k^{n}\right)$ is called invariant if $V$ is closed and invariant under the right $G$-action.

Elek defines the average dimension $\operatorname{dim}_{G}^{\mathcal{A}}(V)$ of an invariant subspace $V$ by choosing a Følner sequence $\left(F_{n}\right)_{n \in \mathbb{N}}$ of $G$ and setting

$$
\operatorname{dim}_{G}^{\mathcal{A}}(V)=\limsup _{n \rightarrow \infty} \frac{\operatorname{dim}_{k}\left(\left.V\right|_{F_{n}}\right)}{\left|F_{n}\right|} .
$$

Theorem 1.8 ([5, Prop. 7.2 and Prop. 9.2]). The sequence in (1.7) converges, and its limit $\operatorname{dim}_{G}^{\mathcal{A}}(V)$ is independent of the choice of the Følner sequence.

Remark 1.9. Elek actually defines $\operatorname{dim}_{G}^{\mathcal{A}}(V)$ using Følner exhaustions, i.e. increasing Følner sequences $\left(F_{n \in \mathbb{N}}\right)$ with $\bigcup_{n \in \mathbb{N}} F_{n}=G$. This makes no difference since the existence of the limit of $\left(\operatorname{dim}_{k}\left(\left.V\right|_{F_{n}}\right) /\left|F_{n}\right|\right)_{n \in \mathbb{N}}$ for arbitrary Følner sequences (and thus its independence of the choice) follows from [9, Theorem 6.1].

Let $M$ be a finitely generated left $k G$-module. The $k$-dual $M^{*}=\operatorname{hom}_{k}(M, k)$ (where $M$ and $k$ are viewed as left $k$-modules, and $(\phi a) m=\phi(a m)$ for $\phi \in M^{*}$, $a \in k$ and $m \in M)$ carries the natural right $G$-action $(\phi g)(m)=\phi(g m)$. The dual of the free left $k G$-module $k G^{n}$ is canonically isomorphic to $\operatorname{map}\left(G, k^{n}\right)$. Any left $k G$-surjection $f: k G^{n} \rightarrow M$ induces a right $k G$-injection $f^{*}: M^{*} \rightarrow \operatorname{map}\left(G, k^{n}\right)$ such that $\operatorname{im}\left(f^{*}\right)$ is a $G$-invariant $k$-subspace.

Definition 1.10 (Elek's dimension function). Let $M$ be a finitely generated left $k G$-module. Its dimension in the sense of Elek is defined by choosing a left $k G$ surjection $f: k G^{n} \rightarrow M$ and setting

$$
\operatorname{dim}_{k G}^{\text {Elek }}(M)=\operatorname{dim}_{G}^{\mathcal{A}}\left(\operatorname{im}\left(f^{*}\right)\right) .
$$

Theorem 1.12 (Main properties of Elek's dimension function). Let $G$ be a finitely generated amenable group. The definition (1.11) of $\operatorname{dim}_{k G}^{\text {Elek }}(M)$ is independent of the choice of the surjection $f$, and $\operatorname{dim}_{k G}^{\text {Elek }}$ has the following properties:

(i) $\operatorname{dim}_{k G}^{\text {Elek }}(k G)=1$.

(ii) For any short exact sequence of finitely generated $k G$-modules $0 \rightarrow M_{0} \rightarrow$ $M_{1} \rightarrow M_{2} \rightarrow 0$ we get

$$
\operatorname{dim}_{k G}^{\text {Elek }}\left(M_{1}\right)=\operatorname{dim}_{k G}^{\text {Elek }}\left(M_{0}\right)+\operatorname{dim}_{k G}^{\text {Elek }}\left(M_{2}\right) .
$$

(iii) If the finitely generated $k G$-module $M$ satisfies $\operatorname{dim}_{k G}^{\text {Elek }}(M)=0$, then every quotient module $Q$ of $M$ satisfies $\operatorname{dim}_{k G}^{\text {Elek }}(Q)=0$.

Proof. The first two assertions are proved in [5, Theorem 1]. Notice that the third condition does not necessarily follow from additivity since the kernel of the epimorphism $M \rightarrow Q$ may not be finitely generated. But the third statement is a direct consequence of the definition of Elek's dimension.

Remark 1.13 (The dual of finitely generated $k G$-modules). Identify the left $k G$ module $k G^{n}$ with the finitely supported functions in $\operatorname{map}\left(G, k^{n}\right)$. Here we view 
$\operatorname{map}\left(G, k^{n}\right)$ as a left $k$-vector space by $(a f)(g)=a f(g)$, and the left $G$-action is given by $(h f)(g)=f\left(h^{-1} g\right)$ for $h, g \in G$ and $a \in k$. Let

$$
\left\langle-_{-},\right\rangle: k G^{n} \times \operatorname{map}\left(G, k^{n}\right)
$$

be the canonical pairing (evaluation) of $k G^{n}$ and its dual $\operatorname{map}\left(G, k^{n}\right)$. If we view an element $f \in k G^{n}$ as a finitely supported function $G \rightarrow k^{n}\left(\operatorname{in} \operatorname{map}\left(G, k^{n}\right)\right)$, then the pairing of $f \in k G^{n}$ with $l \in \operatorname{map}\left(G, k^{n}\right)$ is given by

$$
\langle f, l\rangle=\sum_{g \in G}(f(g), l(g)),
$$

where $\left({ }_{-},{ }_{-}\right)$denotes the standard inner product in $k^{n}$. For a subset $W \subset k G^{n}$ let

$$
W^{\perp}=\left\{f \in \operatorname{map}\left(G, k^{n}\right) \mid\langle x, f\rangle=0 \forall x \in W\right\} .
$$

If $M$ is a finitely generated $k G$-module and $f: k G^{n} \rightarrow M$ is a left $k G$-surjection, then $f^{*}: M^{*} \hookrightarrow \operatorname{map}\left(G, k^{n}\right)$ is a right $k G$-injection and

$$
\operatorname{im}\left(f^{*}\right)=\operatorname{ker}(f)^{\perp} \subseteq \operatorname{map}\left(G, k^{n}\right) .
$$

\section{Approximation for finitely presented $k G$-Modules FOR ELEK'S DIMENSION FUNCTION}

The main result of this section is

Theorem 2.1. Let $G$ be a finitely generated amenable group. Consider a sequence of normal subgroups of finite index

$$
G=G_{0} \supseteq G_{1} \supseteq G_{2} \supseteq \cdots
$$

such that $\bigcap_{n \geq 0} G_{n}=\{1\}$. Then every finitely presented $k G$-module $M$ satisfies

$$
\operatorname{dim}_{k G}^{\text {Elek }}(M)=\lim _{n \rightarrow \infty} \frac{\operatorname{dim}_{k}\left(k \otimes_{k G_{n}} M\right)}{\left[G: G_{n}\right]} .
$$

Its proof needs some preparation.

Throughout, let $G$ be a finitely generated amenable group. For any subset $S \subset G$ let $k[S]$ be the $k$-subspace of $k G$ generated by $S \subset k G$. Let $j[S]: k[S] \rightarrow k[G]$ be the inclusion and $\operatorname{pr}[S]: k G \rightarrow k[S]$ be the projection given by

$$
\operatorname{pr}[S](g)= \begin{cases}g & \text { if } g \in S, \\ 0 & \text { if } g \in G \backslash S .\end{cases}
$$

Theorem 2.2. Let $G$ be a finitely generated amenable group. Let $M$ be a finitely presented left $k G$-module $M$ with a presentation $k G^{r} \stackrel{f}{\rightarrow} k G^{s} \stackrel{p}{\rightarrow} M \rightarrow 0$. For every subset $S \subset G$ we define

$$
M[S]=\operatorname{coker}\left(\operatorname{pr}[S] \circ f \circ j[S]: k[S]^{r} \rightarrow k[S]^{s}\right) .
$$

Let $\left(F_{n}\right)_{n \geq 0}$ be a Følner sequence of $G$. Then

$$
\operatorname{dim}_{k G}^{\text {Elek }}(M)=\lim _{n \rightarrow \infty} \frac{\operatorname{dim}_{k}\left(M\left[F_{n}\right]\right)}{\left|F_{n}\right|} .
$$


Proof. The map $f$ is given by right multiplication with a matrix $A \in M_{r, s}(k G)$. Viewing $A$ as a map $G \rightarrow k^{r \times s}$ it is clear what we mean by the $\operatorname{support} \operatorname{supp}(A)$ of $A$. Let $R>0$ be the diameter of $\operatorname{supp}(A) \cup \operatorname{supp}(A)^{-1}$. Since

$$
\lim _{n \rightarrow \infty} \frac{\left|\partial_{R} F_{n}\right|}{\left|F_{n}\right|}=0
$$

it is enough to show that for every $n \geq 1$

$$
\left|\operatorname{dim}_{k}\left(M\left[F_{n}\right]\right)-\operatorname{dim}_{k}\left(\left.\operatorname{im}\left(p^{*}\right)\right|_{F_{n}}\right)\right| \leq s \cdot\left|\partial_{R} F_{n}\right| .
$$

For the definition of inner products $\left(_{-},{ }_{-}\right)$and $\left\langle_{-},{ }_{-}\right\rangle$we refer to Remark 1.13 . Define the following $k$-linear subspaces of $\operatorname{map}\left(F_{n}, k^{s}\right)$ :

$$
\begin{aligned}
W_{n} & =\left\{\phi: F_{n} \rightarrow k^{s} \mid\left\langle\operatorname{pr}_{n} \circ f \circ j_{n}(x), \phi\right\rangle=0 \forall x \in k\left[F_{n}\right]^{r}\right\}, \\
V_{n} & =\left\{\phi: F_{n} \rightarrow k^{s} \mid \exists \bar{\phi}: G \rightarrow k^{s} \text { satisfying }\left.\bar{\phi}\right|_{F_{n}}=\phi,\langle f(y), \bar{\phi}\rangle=0 \forall y \in k G^{r}\right\}, \\
Z_{n} & =\left\{\phi: F_{n} \rightarrow k^{s}|\phi|_{\partial_{R} F_{n}}=0\right\} .
\end{aligned}
$$

Since $\operatorname{dim}_{k}\left(M\left[F_{n}\right]\right)=\operatorname{dim}_{k}\left(W_{n}\right)$ and $\operatorname{dim}_{k}\left(\left.\operatorname{im}\left(p^{*}\right)\right|_{F_{n}}\right)=\operatorname{dim}_{k}\left(V_{n}\right)$, the desired estimate (2.3) is equivalent to

$$
\left|\operatorname{dim}_{k}\left(W_{n}\right)-\operatorname{dim}_{k}\left(V_{n}\right)\right| \leq s \cdot\left|\partial_{R} F_{n}\right| .
$$

By additivity of $\operatorname{dim}_{k}$ we obtain that

Similarly, we get

$$
\begin{aligned}
\operatorname{dim}_{k}\left(W_{n} \cap Z_{n}\right) & \geq \operatorname{dim}_{k}\left(W_{n}\right)-\operatorname{dim}_{k}\left(\operatorname{map}\left(F_{n}, k^{s}\right)\right)+\operatorname{dim}_{k}\left(Z_{n}\right) \\
& \geq \operatorname{dim}_{k}\left(W_{n}\right)-s \cdot\left|F_{n}\right|+s \cdot\left(\left|F_{n}\right|-\left|\partial_{R} F_{n}\right|\right) \\
& =\operatorname{dim}_{k}\left(W_{n}\right)-s \cdot\left|\partial_{R} F_{n}\right| .
\end{aligned}
$$

$$
\operatorname{dim}_{k}\left(V_{n} \cap Z_{n}\right) \geq \operatorname{dim}_{k}\left(V_{n}\right)-s \cdot\left|\partial_{R} F_{n}\right| .
$$

To prove (2.4) it hence suffices to show that

$$
\begin{gathered}
W_{n} \cap Z_{n} \subset V_{n}, \\
V_{n} \cap Z_{n} \subset W_{n} .
\end{gathered}
$$

Let $\phi \in W_{n} \cap Z_{n}$. Extend $\phi$ by zero to a function $\bar{\phi}: G \rightarrow k^{s}$. Let $y \in k G^{r}$. Then we can decompose $y$ as $y=y_{0}+y_{1}$ with $\operatorname{supp}\left(y_{0}\right) \subset F_{n}$ and $\operatorname{supp}\left(y_{1}\right) \subset G \backslash F_{n}$. By definition of the radius $R$ it is clear that $\operatorname{supp}\left(f\left(y_{1}\right)\right) \subset G \backslash F_{n} \cup \partial_{R} F_{n}$. Because of $\phi \in Z_{n}$ we have $\left\langle f\left(y_{1}\right), \bar{\phi}\right\rangle=0$. The fact that $\phi \in W_{n}$ implies that

$$
\left\langle f\left(y_{0}\right), \bar{\phi}\right\rangle=\left\langle\operatorname{pr}_{n} \circ f \circ j_{n}\left(y_{0}\right), \phi\right\rangle=0 .
$$

So we obtain that $\langle f(y), \bar{\phi}\rangle=0$, meaning that $\phi \in V_{n}$. The proof of (2.6) is similar.

The following theorem is due to Weiss. Its proof can be found in [2, Proposition 5.5].

Theorem 2.7 (Weiss). Let $G$ be a countable amenable group. Let $G_{n} \subset G, n \geq 1$, be a sequence of normal subgroups of finite index with $\bigcap_{n>1} G_{n}=\{1\}$. Then there exists, for every $R \geq 1$ and every $\epsilon>0$, an integer $M=M(R, \epsilon) \geq 1$ such that for $n \geq M$ there is a fundamental domain $Q_{n} \subset G$ of the coset space $G / G_{n}$ such that

$$
\frac{\left|\partial_{R} Q_{n}\right|}{\left|Q_{n}\right|}<\epsilon
$$


Now we are ready to prove Theorem 2.1

Proof of Theorem 2.1. According to Theorem 2.7let $\left(Q_{n}\right)_{n \geq 0}$ be a Følner sequence of $G$ such that $Q_{n} \subset G$ is a fundamental domain for $G / G_{n}$. Choose a finite presentation of $M$ :

$$
k G^{r} \stackrel{f}{\rightarrow} k G^{s} \rightarrow M \rightarrow 0 .
$$

Let $f_{n}=k\left[G / G_{n}\right] \otimes_{k G} f$. By right-exactness of tensor products we have the exact sequence

$$
k\left[G / G_{n}\right]^{r} \stackrel{f_{n}}{\longrightarrow} k\left[G / G_{n}\right]^{s} \rightarrow k\left[G / G_{n}\right] \otimes_{k G} M \rightarrow 0 .
$$

The natural map $Q_{n} \subset G \rightarrow G / G_{n}$ induces an isomorphism $j_{n}: k\left[Q_{n}\right] \rightarrow k\left[G / G_{n}\right]$ of $k$-vector spaces. The map $f$ is given by right multiplication $f=R_{A}$ with a matrix $A \in M_{r, s}(k G)$. Viewing $A$ as a map $G \rightarrow k^{r \times s}$, let $\operatorname{supp}(A)$ be the support of $A$. Let $R>0$ be the diameter of $\operatorname{supp}(A) \cup \operatorname{supp}(A)^{-1}$ (with respect to the fixed word metric on $G$ ). Then $f$ restricts to a map

$$
\left.f\right|_{Q_{n} \backslash \partial_{R} Q_{n}}: k\left[Q_{n} \backslash \partial_{R} Q_{n}\right]^{r} \rightarrow k\left[Q_{n}\right]^{s} .
$$

Hence there is precisely one $k$-linear map $g$ for which the following diagram of $k$-vector spaces commutes:

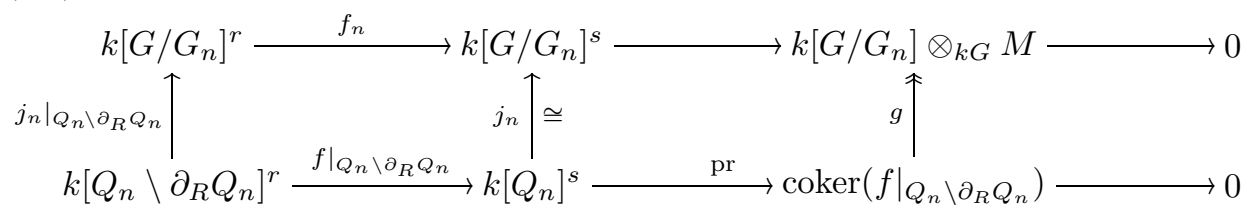

One easily verifies that $g$ is surjective and that

$$
\operatorname{ker}(g) \subset \operatorname{im}\left(\operatorname{pr} \circ j_{n}^{-1} \circ f_{n}: k\left[G / G_{n}\right]^{r} \rightarrow \operatorname{coker}\left(\left.f\right|_{Q_{n} \backslash \partial_{R} Q_{n}}\right)\right) .
$$

The map $\operatorname{pr} \circ j_{n}^{-1} \circ f_{n}$ descends to a map

$$
\operatorname{pr} \circ j_{n}^{-1} \circ f_{n}: \operatorname{coker}\left(\left.j_{n}\right|_{Q_{n} \backslash \partial_{R} Q_{n}}\right) \rightarrow \operatorname{coker}\left(\left.f\right|_{Q_{n} \backslash \partial_{R} Q_{n}}\right) .
$$

Note that

$$
\operatorname{dim}_{k}\left(\operatorname{coker}\left(\left.j_{n}\right|_{Q_{n} \backslash \partial_{R} Q_{n}}\right)\right)=r \cdot\left|\partial_{R} Q_{n}\right|
$$

Thus,

$$
\operatorname{dim}_{k}\left(\operatorname{coker}\left(\left.f\right|_{Q_{n} \backslash \partial_{R} Q_{n}}\right)\right)-\operatorname{dim}_{k}\left(k\left[G / G_{n}\right] \otimes_{k G} M\right)=\operatorname{dim}_{k} \operatorname{ker}(g) \leq r \cdot\left|\partial_{R} Q_{n}\right| .
$$

By replacing the upper row in diagram (2.8) by

$$
k\left[Q_{n}\right]^{r} \stackrel{\operatorname{pr}\left[Q_{n}\right] \circ f \circ j\left[Q_{n}\right]}{\longrightarrow} k\left[Q_{n}\right]^{s} \rightarrow M\left[Q_{n}\right] \rightarrow 0
$$

and essentially running the same argument as before, we obtain that

$$
\operatorname{dim}_{k}\left(\operatorname{coker}\left(\left.f\right|_{Q_{n} \backslash \partial_{R} Q_{n}}\right)\right)-\operatorname{dim}_{k}\left(\operatorname{coker}\left(M\left[Q_{n}\right]\right)\right) \leq r \cdot\left|\partial_{R} Q_{n}\right| \cdot
$$

Since

we get that

$$
\frac{\left|\partial_{R} Q_{n}\right|}{\left[G / G_{n}\right]}=\frac{\left|\partial_{R} Q_{n}\right|}{\left|Q_{n}\right|} \stackrel{n \rightarrow \infty}{\longrightarrow} 0
$$

$$
\lim _{n \rightarrow \infty} \frac{\operatorname{dim}_{k}\left(k\left[G / G_{n}\right] \otimes_{k G} M\right)}{\left[G: G_{n}\right]}
$$


exists if and only if

$$
\lim _{n \rightarrow \infty} \frac{\operatorname{dim}_{k}\left(M\left[Q_{n}\right]\right)}{\left|Q_{n}\right|}
$$

exists, and in this case they are equal. Now the assertion follows from Theorem 2.2

\section{COMPARING DIMENSIONS}

The main result of this section is

Theorem 3.1 (Comparing dimensions). Let $G$ be a group, let $k$ be a skew field, and let $k * G$ be a crossed product which is prime left Goldie. Let dim be any dimension function which assigns to a finitely generated left $k * G$-module a non-negative real number and satisfies

(i) $\operatorname{dim}(k * G)=1$.

(ii) For every short exact sequence $0 \rightarrow M_{0} \rightarrow M_{1} \rightarrow M_{2} \rightarrow 0$ of finitely generated left $k * G$-modules, we get

$$
\operatorname{dim}\left(M_{1}\right)=\operatorname{dim}\left(M_{0}\right)+\operatorname{dim}\left(M_{2}\right) .
$$

(iii) If the finitely generated left $k * G$-module $M$ satisfies $\operatorname{dim}(M)=0$, then every quotient module $Q$ of $M$ satisfies $\operatorname{dim}(Q)=0$.

Then for every finitely presented left $k * G$-module $M$, we get

$$
\operatorname{dim}(M)=\operatorname{dim}_{k * G}^{\text {Ore }}(M) .
$$

Proof. Let $S$ denote the non-zero-divisors of $k * G$. We have to show that for all $r, s \in \mathbb{N}$ and every $r \times s$ matrix $A$ with entries in $k * G$

$\operatorname{dim}_{k * G}^{\text {Ore }}\left(\operatorname{coker}\left(r_{A}: S^{-1} k * G^{r} \rightarrow S^{-1} k * G^{s}\right)\right)=\operatorname{dim}\left(\operatorname{coker}\left(r_{A}: k * G^{r} \rightarrow k * G^{s}\right)\right)$,

where $r_{A}$ denotes the module homomorphism given by right multiplication with $A$. First note that we may assume that $r=s$. Indeed if $r<s$, replace $A$ with the $s \times s$ matrix which is $A$ for the first $r$ rows and has 0 's on the bottom $s-r$ rows. On the other hand if $r>s$, replace $A$ with the $r \times r$ matrix $B$ with entries $\left(b_{i j}\right)$ which is $A$ for the first $s$ columns and has $b_{i j}=\delta_{i j}$ if $i>s$, where $\delta_{i j}$ is the Kronecker delta.

We will often use the obvious long exact sequence associated to homomorphisms $f: M_{0} \rightarrow M_{1}$ and $g: M_{1} \rightarrow M_{2}$ :

$$
0 \rightarrow \operatorname{ker}(f) \rightarrow \operatorname{ker}(g \circ f) \rightarrow \operatorname{ker}(g) \rightarrow \operatorname{coker}(f) \rightarrow \operatorname{coker}(g \circ f) \rightarrow \operatorname{coker}(g) \rightarrow 0 .
$$

We now assume that $A$ is an $r \times r$ matrix. Note that equation (3.2) is true if $A$ is invertible over $S^{-1} k * G$; this is because then $\operatorname{ker} r_{A}=0$ (whether $A$ is considered as a matrix over $k * G$ or $\left.S^{-1} k * G\right)$.

Next observe that if $U \in \mathrm{M}_{r}(k * G)$ which is invertible over $\mathrm{M}_{r}\left(S^{-1} k * G\right)$, then equation (3.2) holds for $A$ if and only if it holds for $A U$, and also if and only if it holds for $U A$. This follows from (3.3), $\operatorname{ker} U=0, \operatorname{dim}(\operatorname{coker} U)=\operatorname{dim}_{k * G}^{\text {Ore }}(\operatorname{coker} U)=0$, and in the second case we use the third property of dim.

We may write $S^{-1} k * G=\mathrm{M}_{d}(D)$ for some $d \in \mathbb{N}$ and some skew field $D$. By applying the Morita equivalence from $\mathrm{M}_{d}(D)$ to $D$ and back and by doing Gaussian elimination over $D$ we see that there are invertible matrices $U, V \in \mathrm{M}_{r d}\left(S^{-1} k * G\right)$ such that $U \operatorname{diag}(A, \ldots, A) V=J$, where there are $d A$ 's and $J$ is a matrix of the 
form $\operatorname{diag}(1, \ldots, 1,0, \ldots, 0)$. Now choose $u, v \in S$ such that $u U, v V \in \mathrm{M}_{r d}(k * G)$. Then $(u U) \operatorname{diag}(A, \ldots, A)(V v)=u J v$, and the result follows.

Theorem 3.4 (Comparing Elek's dimension and the Ore dimension). Let $G$ be a finitely generated group and let $k$ be a skew field. Suppose that $k G$ is a prime left Goldie ring. Then for any finitely presented left $k G$-module $M$,

$$
\operatorname{dim}_{k G}^{\text {Elek }}(M)=\operatorname{dim}_{k G}^{\text {Ore }}(M) .
$$

Proof. This follows from Theorem 3.1 and Theorem 1.12

\section{Proof of the main theorem}

Proof of Theorem 0.2, (i) In the first step we reduce the claim to the case where $G$ is finitely generated. Consider a finitely presented left $k G$-module $M$. Choose a matrix $A \in M_{r, s}(k G)$ such that $M$ is $k G$-isomorphic to the cokernel of $r_{A}: k G^{r} \rightarrow$ $k G^{s}$. Since $A$ is a finite matrix and each element in $k G$ has finite support, we can find a finitely generated subgroup $H \subseteq G$ such that $A \in M_{r, s}(k H)$. Both $k G$ and $k H$ are prime left Goldie by Lemma 1.4 and Theorem 1.6. Consider the finitely presented $k H$-module $N:=\operatorname{coker}\left(r_{A}: k H^{r} \rightarrow k H^{s}\right)$. Then $M=k G \otimes_{k H} N$. We can also consider the Ore localization $T^{-1} k H$ for $T$ the set of non-zero-divisors of $k H$. Put $H_{n}=H \cap G_{n}$. We obtain a residual chain $\left(H_{n}\right)_{n \geq 0}$ of $H$ and have

$$
\begin{aligned}
\operatorname{dim}_{k G}^{\text {Ore }}(M) & =\operatorname{dim}_{S^{-1} k G}\left(S^{-1} k G \otimes_{k G} M\right) \\
& =\operatorname{dim}_{S^{-1} k G}\left(S^{-1} k G \otimes_{k G} k G \otimes_{k H} N\right) \\
& =\operatorname{dim}_{S^{-1} k G}\left(S^{-1} k G \otimes_{T^{-1} k H} T^{-1} k H \otimes_{k H} N\right) \\
& =\operatorname{dim}_{T^{-1} k H}\left(T^{-1} k H \otimes_{k H} N\right) \\
& =\operatorname{dim}_{k H}^{\text {Ore }}(N) .
\end{aligned}
$$

We compute

$$
\begin{aligned}
\frac{\operatorname{dim}_{k}\left(k \otimes_{k G_{n}} M\right)}{\left[G: G_{n}\right]} & =\frac{\operatorname{dim}_{k}\left(k\left[G / G_{n}\right] \otimes_{k G} M\right)}{\left[G: G_{n}\right]} \\
& =\frac{\operatorname{dim}_{k}\left(k\left[G / G_{n}\right] \otimes_{k G} k G \otimes_{k H} N\right)}{\left[G: G_{n}\right]} \\
& =\frac{\operatorname{dim}_{k}\left(k\left[G / G_{n}\right] \otimes_{k\left[H / H_{n}\right]} k\left[H / H_{n}\right] \otimes_{k H} N\right)}{\left[G: G_{n}\right]} \\
& =\frac{\left[G / G_{n}: H / H_{n}\right] \cdot \operatorname{dim}_{k}\left(k\left[H / H_{n}\right] \otimes_{k H} N\right)}{\left[G: G_{n}\right]} \\
& =\frac{\left[G / G_{n}: H / H_{n}\right] \cdot \operatorname{dim}_{k}\left(k \otimes_{k H_{n}} N\right)}{\left[G / G_{n}: H / H_{n}\right] \cdot\left[H: H_{n}\right]} \\
& =\frac{\operatorname{dim}_{k}\left(k \otimes_{k H_{n}} N\right)}{\left[H: H_{n}\right]} .
\end{aligned}
$$

Therefore the claim holds for $M$ over $k G$ if it holds for $N$ over $k H$. Hence we can assume without loss of generality that $G$ is finitely generated.

Now apply Theorem 2.1 and Theorem 3.4 . 
(ii) We obtain from additivity, the exactness of the functor $S^{-1} k G \otimes_{k G}$ - and the right exactness of the functor $k \otimes_{k G}$ - that

$$
\begin{aligned}
\operatorname{dim}_{k G}^{\text {Ore }}\left(H_{i}\left(C_{*}\right)\right)= & \operatorname{dim}_{k G}^{\text {Ore }}\left(\operatorname{coker}\left(c_{i+1}\right)\right)+\operatorname{dim}_{k G}^{\text {Ore }}\left(\operatorname{coker}\left(c_{i}\right)\right)-\operatorname{dim}_{k G}^{\text {Ore }}\left(C_{i-1}\right), \\
\operatorname{dim}_{k}\left(H_{i}\left(k \otimes_{k G_{n}} C_{*}\right)\right)=\operatorname{dim}_{k}\left(k \otimes_{k G_{n}} \operatorname{coker}\left(c_{i+1}\right)\right)+\operatorname{dim}_{k}\left(k \otimes_{k G_{n}} \operatorname{coker}\left(c_{i}\right)\right) & -\operatorname{dim}_{k}\left(k \otimes_{k G_{n}} C_{i-1}\right) .
\end{aligned}
$$

Hence the claim follows from assertion (i) applied to the finitely presented $k G$ modules coker $\left(c_{i+1}\right)$, coker $\left(c_{i}\right)$ and $C_{i-1}$.

(iii) This follows from assertion (ii) applied to the cellular chain complex of $X$.

\section{Extension to THE VIRTUALLY TORSIONFREE CASE}

Next we explain how Theorem 0.2 can be extended to the virtually torsionfree case.

For the remainder of this section let $k$ be a skew field, let $G$ be an amenable group which possesses a subgroup $H$ of finite index with $\Delta^{+}(H)=1$, and let $k * G$ be a crossed product such that $k * H$ is a left Goldie ring. We define the virtual Ore dimension of a $k * G$-module $M$ by

$$
\operatorname{vdim}_{k * G}^{\text {Ore }}(M)=\frac{\operatorname{dim}_{k * H}^{\text {Ore }}\left(\operatorname{res}_{k * G}^{k * H} M\right)}{[G: H]},
$$

where $\operatorname{res}_{k * G}^{k * H} M$ is the $k * H$-module obtained from the $k * G$-module $M$ by restricting the $G$-action to $H$.

We have to show that this is independent of the choice of $H$. Since every subgroup of finite index contains a normal subgroup of finite index, it is enough to show that if $K$ is a normal subgroup of finite index in $H$ and $K \leq H \leq G$ with $H$ torsion free, then for every $k * H$-module $N$,

$$
\frac{\operatorname{dim}_{k * K}^{\text {Ore }}\left(\operatorname{res}_{k * H}^{k * K} N\right)}{[H: K]}=\operatorname{dim}_{k * H}^{\text {Ore }}(N) .
$$

Let $T$ denote the set of non-zero-divisors of $k * H$ and write $S=(k * K) \cap T$. Note that $\Delta^{+}(K)=1$, so $k * K$ is still a prime left Goldie ring and hence the ring $S^{-1} k * K$ exists. Then $S^{-1} k * H \cong\left(S^{-1} k * K\right) *[H / K]$ and there is a natural ring monomorphism $\theta: S^{-1} k * H \hookrightarrow T^{-1} k * H$. Since $S^{-1} k * K$ is a matrix ring over a skew field by Theorem [1.5, we see that $\left(S^{-1} k * K\right)[H / K]$ is an Artinian ring because $H / K$ is finite. But every element of $T$ is a non-zero-divisor in $\left(S^{-1} k * K\right)[H / K]$, and since every non-zero-divisor in an Artinian ring is invertible (compare [16, Exercise 22 of Chapter 15 on p. 16]), we see that every element of $T$ is invertible in $S^{-1} k * H$ and we conclude that $\theta$ is onto and hence is an isomorphism. We deduce that $\operatorname{dim}_{S^{-1} k * K}\left(T^{-1} k * H\right)=[H: K]$ and that the natural map $S^{-1} N \rightarrow T^{-1} N$ induced by $s^{-1} n \mapsto s^{-1} n$ is an isomorphism. This proves (5.2).

Theorem 5.3 (Extension to the virtually torsionfree case). Let $G$ be an amenable group which possesses a subgroup $E \subseteq G$ of finite index such that $k E$ is left Goldie and $\Delta^{+}(E)=1$, and let $k$ be a skew field. Then assertions (i), (ii)] and (iii) of Theorem 0.2 remain true, provided we replace $\operatorname{dim}_{k G}^{\text {Ore by }}$ vdim $\operatorname{dim}_{k}^{\text {Ore everywhere. }}$ 
Proof. It suffices to prove the claim for assertion (i) since the proof in Theorem 0.2 that it implies the other two assertions applies also in this more general situation. Let $\left(G_{n}\right)_{n \geq 0}$ be a residual chain of $G$. To prove the result in general, we may assume that $G$ is finitely generated. Since $k E$ is left Goldie and $[G: E]<\infty$, the group ring $k G$ is also left Goldie. Further, every $k G_{n}$ is left Goldie. Since $\Delta^{+}(G)$ is finite (its order is bounded by $[G: E]$ ), there exists $N \in \mathbb{N}$ such that $G_{N} \cap \Delta^{+}(G)=1$, and then $\Delta^{+}\left(G_{N}\right)=1$ and $G_{i} \subseteq G_{N}$ for all $i \geq N$. Set $H=G_{N}$ so that $k H$ is prime by Lemma 1.4. Then for a finitely presented $k H$-module $L$,

$$
\operatorname{dim}_{k H}^{\mathrm{Ore}}(L)=\lim _{n \rightarrow \infty} \frac{\operatorname{dim}_{k}\left(k \otimes_{k\left[G_{n} \cap H\right]} L\right)}{\left[H: H \cap G_{n}\right]}
$$

by Theorems 2.1 and 3.1. We have $\left[G: G_{n}\right]=[G: H] \cdot\left[H: H \cap G_{n}\right]$ for $n \geq N$. This implies for every finitely presented $k G$-module $M$

$$
\begin{aligned}
\operatorname{vdim}_{k G}^{\text {Ore }}(M)=\frac{\operatorname{dim}_{k H}^{\text {Ore }}\left(\operatorname{res}_{k G}^{k H} M\right)}{[G: H]} & =\lim _{n \rightarrow \infty} \frac{\operatorname{dim}_{k}\left(k \otimes_{k\left[H \cap G_{n}\right]} \operatorname{res}_{k G}^{k H} M\right)}{[G: H] \cdot\left[H: H \cap G_{n}\right]} \\
& =\lim _{n \rightarrow \infty} \frac{\operatorname{dim}_{k}\left(k \otimes_{k G_{n}} M\right)}{\left[G: G_{n}\right]} .
\end{aligned}
$$

Remark 5.4. Because of Theorem 1.6, Theorem 0.2 is true in the case where $k$ is a skew field and $G$ is an elementary amenable group in which the orders of the finite subgroups are bounded (clearly $\Delta^{+}\left(G_{n}\right)=1$ for sufficiently large $n$ ). In particular Theorem 0.2 is true for any virtually torsionfree elementary amenable group.

\section{EXAMPLES}

Remark 6.1. Let $\left(G_{n}\right)_{n \geq 0}$ be a residual chain of a group $G$. Let $X$ be a finite free $G-C W$-complex. Let $k$ be a field of characteristic $\operatorname{char}(k)$. For a prime $p$ denote by $\mathbb{F}_{p}$ the field of $p$ elements. Then we conclude from the universal coefficient theorem that

$$
\begin{aligned}
& \operatorname{dim}_{k}\left(H_{i}\left(G_{n} \backslash X ; k\right)\right)=\operatorname{dim}_{\mathbb{Q}}\left(H_{i}\left(G_{n} \backslash X ; \mathbb{Q}\right)\right) \quad \operatorname{char}(k)=0, \\
& \operatorname{dim}_{k}\left(H_{i}\left(G_{n} \backslash X ; k\right)\right)=\operatorname{dim}_{\mathbb{F}_{p}}\left(H_{i}\left(G_{n} \backslash X ; \mathbb{F}_{p}\right)\right) \quad p=\operatorname{char}(k) \neq 0, \\
& \operatorname{dim}_{\mathbb{F}_{p}}\left(H_{i}\left(G_{n} \backslash X ; \mathbb{F}_{p}\right)\right) \geq \operatorname{dim}_{\mathbb{Q}}\left(H_{i}\left(G_{n} \backslash X ; \mathbb{Q}\right)\right) .
\end{aligned}
$$

In particular we conclude from Remark 0.3 that

$$
\liminf _{n \rightarrow \infty} \frac{\operatorname{dim}_{k}\left(H_{i}\left(G_{n} \backslash X ; k\right)\right)}{\left[G: G_{n}\right]} \geq \lim _{n \rightarrow \infty} \frac{\operatorname{dim}_{k}\left(H_{i}\left(G_{n} \backslash X ; \mathbb{Q}\right)\right)}{\left[G: G_{n}\right]}=b_{i}^{(2)}(X ; \mathcal{N}(G)),
$$

where the latter term denotes the $i$-th $L^{2}$-Betti number of $X$. In particular we get from Theorem 0.2 for a torsionfree amenable group $G$ with no zero-divisors in $k G$ that

$$
\begin{aligned}
\operatorname{dim}_{k G}^{\text {Ore }}\left(H_{i}(X ; k)\right)=\lim _{n \rightarrow \infty} \frac{\operatorname{dim}_{k}\left(H_{i}\left(G_{n} \backslash X ; k\right)\right)}{\left[G: G_{n}\right]} & \geq \lim _{n \rightarrow \infty} \frac{\operatorname{dim}_{k}\left(H_{i}\left(G_{n} \backslash X ; \mathbb{Q}\right)\right)}{\left[G: G_{n}\right]} \\
& =b_{i}^{(2)}(X ; \mathcal{N}(G)) \\
& =\operatorname{dim}_{\mathbb{C} G}^{\text {Ore }}\left(H_{i}(X ; \mathbb{C})\right) .
\end{aligned}
$$

This inequality is in general not an equality, as the next example shows. 
Example 6.2. Fix an integer $d \geq 2$ and a prime number $p$. Let $f_{p}: S^{d} \rightarrow S^{d}$ be a map of degree $p$ and denote by $i: S^{d} \rightarrow S^{1} \vee S^{d}$ the obvious inclusion. Let $X$ be the finite $C W$-complex obtained from $S^{1} \vee S^{d}$ by attaching a $(d+1)$-cell with an attaching map $i \circ f^{d}: S^{d} \rightarrow S^{1} \vee S^{d}$. Then $\pi_{1}(X)=\mathbb{Z}$. Let $\widetilde{X}$ be the universal covering of $X$ which is a finite free $\mathbb{Z}-C W$-complex. Denote by $X_{n}$ the covering of $X$ associated to $n \cdot \mathbb{Z} \subseteq \mathbb{Z}$. The cellular $\mathbb{Z} C$-chain complex of $\widetilde{X}$ is concentrated in dimension $(d+1), d$ and 1 and 0 , the $(d+1)$-th differential is multiplication with $p$ and the first differential is multiplication with $(z-1)$ for a generator $z \in \mathbb{Z}$ :

$$
0 \rightarrow \cdots \rightarrow \mathbb{Z}[\mathbb{Z}] \stackrel{p}{\rightarrow} \mathbb{Z}[\mathbb{Z}] \rightarrow \cdots \rightarrow \mathbb{Z}[\mathbb{Z}] \stackrel{z-1}{\rightarrow} \mathbb{Z}[\mathbb{Z}] .
$$

If the characteristic of $k$ is different from $p$, one easily checks that $H_{i}\left(C_{*}\right)=0$ and

$$
\operatorname{dim}_{k \mathbb{Z}}^{\text {Ore }}\left(H_{i}(\widetilde{X} ; k)\right)=0 \text { for } i \in\{d, d+1\} .
$$

If $p$ is the characteristic of $k$, then $H_{i}\left(C_{*}\right)=k \mathbb{Z}$ and

$$
\operatorname{dim}_{k \mathbb{Z}}^{\text {Ore }}\left(H_{i}(\tilde{X} ; k)\right)=1 \text { for } i \in\{d, d+1\} .
$$

Hence $\operatorname{dim}_{k G}^{\text {Ore }}\left(H_{i}(\tilde{X} ; k)\right)$ does depend on $k$ in general.

Example 6.3. Let $G$ be a torsionfree amenable group such that $k G$ has no zerodivisors. Let $S^{1} \rightarrow X \rightarrow B$ be a fibration of connected $C W$-complexes such that $X$ has fundamental group $\pi_{1}(X) \cong G$ and $\pi_{1}\left(S^{1}\right) \rightarrow \pi_{1}(X)$ is injective. Then

$$
\operatorname{dim}_{k G}^{\text {Ore }}\left(H_{i}(\tilde{X} ; k)\right)=0
$$

for every $i \geq 0$.

Let $S=k G-\{0\}$ and $S_{0}=k \mathbb{Z}-\{0\}$. By looking at the cellular chain complex one directly sees that

$$
H_{i}\left(\widetilde{S}^{1}, S_{0}^{-1} k \mathbb{Z}\right)=0 \forall i \geq 0 .
$$

Thus $H_{i}\left(\widetilde{S}^{1}, S^{-1} k G\right)=S^{-1} k G \otimes_{S_{0}^{-1} k \mathbb{Z}} H_{i}\left(\widetilde{S}^{1}, S_{0}^{-1} k \mathbb{Z}\right)=0$ for every $i \geq 0$. The assertion is implied by the Hochschild-Serre spectral sequence that converges to $H_{p+q}\left(\widetilde{X}, S^{-1} k G\right)$ and has the $E^{2}$-term:

$$
E_{p q}^{2}=H_{p}\left(\widetilde{B}, H_{q}\left(\widetilde{S}^{1}, S^{-1} k G\right)\right) .
$$

Example 6.5 (Sublinear growth of Betti numbers). Let $G$ be an infinite amenable group which possesses a subgroup $H$ of finite index such that $k H$ is left Goldie and $\Delta^{+}(H)=1$, e.g., $G$ is a virtually torsionfree elementary amenable group. Let $k$ be a field. Let $\left(G_{n}\right)_{n \geq 0}$ be a residual chain of $G$. Denote by $b_{i}\left(G / G_{n} ; K\right)$ the $i$-th Betti number of the group $G / G_{n}$ with coefficients in $k$. Then we get for every $i \geq 0$

$$
\lim _{n \rightarrow \infty} \frac{b_{i}\left(G / G_{n} ; k\right)}{\left[G: G_{n}\right]}=0 .
$$

For $i=0$ this is obvious. For $i \geq 1$ this follows from Theorem 5.3 and $H_{i}(E H ; k)=$ $H_{i}(H ; k)=0$.

\section{REFERENCES}

[1] M. Abert, A. Jaikin-Zapirain, and N. Nikolov, The rank gradient from a combinatorial viewpoint, 2007. arXiv:math/0701925v2.

[2] C. Deninger and K. Schmidt, Expansive algebraic actions of discrete residually finite amenable groups and their entropy, Ergodic Theory Dynam. Systems 27 (2007), no. 3, 769786. MR2322178 (2008d:37009) 
[3] J. Dodziuk, P. Linnell, V. Mathai, T. Schick, and S. Yates, Approximating $L^{2}$-invariants and the Atiyah conjecture, Comm. Pure Appl. Math. 56 (2003), no. 7, 839-873. Dedicated to the memory of Jürgen K. Moser. MR.1990479 (2004g:58040)

[4] G. Elek, Amenable groups, topological entropy and Betti numbers, Israel J. Math. 132 (2002), 315-335. MR1952628 (2003k:37026)

[5] _ The rank of finitely generated modules over group algebras, Proc. Amer. Math. Soc. 131 (2003), no. 11, 3477-3485 (electronic). MR.1991759 (2004i:43003)

[6] _ The strong approximation conjecture holds for amenable groups, J. Funct. Anal. 239 (2006), no. 1, 345-355. MR2258227 (2007m:43001)

[7] P. H. Kropholler, P. A. Linnell, and J. A. Moody, Applications of a new K-theoretic theorem to soluble group rings, Proc. Amer. Math. Soc. 104 (1988), no. 3, 675-684. MR89j:16016

[8] S. Lang, Algebra, third edition, Graduate Texts in Mathematics, vol. 211, Springer-Verlag, New York, 2002. MR1878556 (2003e:00003)

[9] E. Lindenstrauss and B. Weiss, Mean topological dimension, Israel J. Math. 115 (2000), 1-24. MR:1749670 (2000m:37018)

[10] P. A. Linnell, Noncommutative localization in group rings, Non-commutative localization in algebra and topology, Cambridge Univ. Press, Cambridge, 2006, pp. 40-59. MR2222481 (2007f:16064)

[11] W. Lück, Approximating $L^{2}$-invariants by their finite-dimensional analogues, Geom. Funct. Anal. 4 (1994), no. 4, 455-481. MR.95g:58234

[12] _ $L^{2}$-Invariants: Theory and Applications to Geometry and K-Theory, Ergebnisse der Mathematik und ihrer Grenzgebiete. 3. Folge. A Series of Modern Surveys in Mathematics [Results in Mathematics and Related Areas. 3rd Series. A Series of Modern Surveys in Mathematics], vol. 44, Springer-Verlag, Berlin, 2002. MR,1926 649

[13] D. Pape, A short proof of the approximation conjecture for amenable groups, J. Funct. Anal. 255 (2008), no. 5, 1102-1106. MR2455493 (2010a:46171)

[14] D. S. Passman, The algebraic structure of group rings, Pure and Applied Mathematics, WileyInterscience [John Wiley \& Sons], New York, 1977. MR81d:16001

[15] _ Group rings, crossed products and Galois theory, CBMS Regional Conference Series in Mathematics, vol. 64, published for the Conference Board of the Mathematical Sciences, Washington, DC, by the Amer. Math. Soc., Providence, RI, 1986. MR840467 (87e:16033)

[16] L. H. Rowen, Graduate algebra: Noncommutative view, Graduate Studies in Mathematics, vol. 91, American Mathematical Society, Providence, RI, 2008. MR 2462400 (2009k:16001)

[17] B. Stenström, Rings of quotients. Die Grundlehren der Mathematischen Wissenschaften, Band 217, An introduction to methods of ring theory, Springer-Verlag, New York, 1975. MR.52:10782

[18] B. Weiss, Monotileable amenable groups, Topology, ergodic theory, real algebraic geometry, Amer. Math. Soc. Transl. Ser. 2, 202, Amer. Math. Soc., Providence, RI, 2001, pp. 257-262. MR:1819193 (2001m:22014)

Department of Mathematics, Virginia Tech, Blacksburg, Virginia 24061-0123

E-mail address: plinnell@math.vt.edu

URL: http://www.math.vt.edu/people/plinnell/

Mathematisches Institut, Westfälische Wilhelms-Universität Münster, EinsteinStrasse 62, D-48149 Münster, Germany

E-mail address: lueck@math.uni-muenster.de

$U R L:$ http://www.math.uni-muenster.de/u/lueck

Mathematisches Institut, Westfälische Wilhelms-Universität Münster, EinsteinStrasse 62, D-48149 Münster, Germany

E-mail address: sauerr@uni-muenster.de

$U R L:$ http://wwwmath.uni-muenster.de/u/sauerr/ 